\title{
Hilft Botox gegen Depressionen?
}

40-60\% der Epileptiker leiden auch unter schweren Depressionen. In einer randomisierten, doppelt kontrollierten Pilotstudie wurde von Forschern der Universität Basel und der Medizinischen Hochschule Hannover untersucht, inwieweit Botoxinjektionen in die Stirnregion diese Depressionen, die nur schwer mit Antidepressiva zu beeinflussen waren, besserten.

- An der Studie nahmen 30 Patienten teil. 15 der Patienten erhielten Botoxinjektionen in die Stirn, die anderen bekamen eine Placeboinjektion (Saline).

Die Patienten der Botoxgruppe waren schon nach zwei Wochen weniger depressiv, Die Schwere der Symptome hatte sich bei $60 \%$ nach sechs Wochen mindestens halbiert $(\mathrm{p}<0,002)$. Bis zum Ende der Studie nach 16 Wochen fühlten sich die Probanden sogar noch wohler $(47,1 \%)$. In der Placebo-Gruppe besserten sich die Symptome dagegen nur geringfügig $(9,2 \%)$.

\section{Kommentar}

Das Nervengift Botox glättet nicht nur Stirnfalten. Es kann vielleicht sogar Depressionen lindern. Die Behandlung ist relativ nebenwirkungsarm, sicher und ökonomisch, da die Wirkung einer Spritze mehrere Monate anhält. Botulinumtoxin könnte ein neuer Bestandteil in der Depressionsbehandlung werden Bevor es aber breit eingesetzt wird, sollte noch in einer größeren Patientengruppe geklärt werden, unter welchen Bedingungen Botox Depressionen lindern kann und wie sich dies genau abspielt.

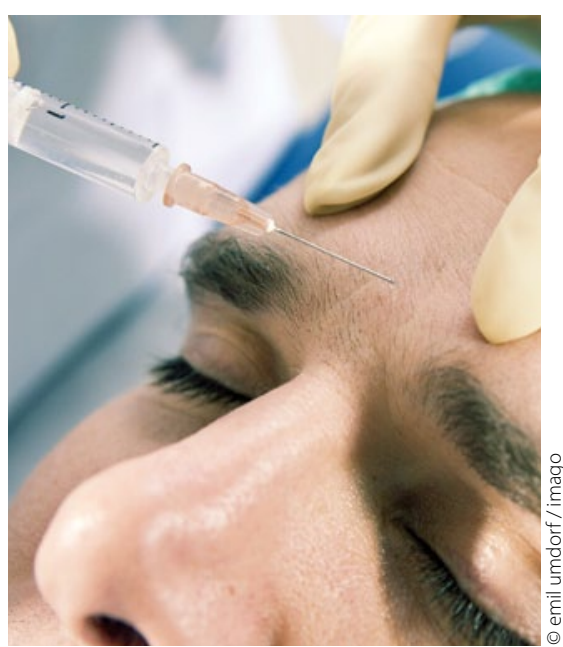

Botox, wenn Antidepressiva versagen?

- M. A. Wollmer et al.

Facing depression with botulinum toxin: A randomized controlled trial. Published online on 23 February 2012 in J Psychiatr. Res.; doi: org. 10. 1016/j.psychires.2012.01.027.

\section{Nächtliche Wadenkrämpfe durch Medikamente}

Langwirksame Beta-2-Sympathikomimetika, Diuretika und Statine erhöhen das Risiko für nächtliche Wadenkrämpfe.

- In Kanada wurde geprüft, wie Chinin gegen nächtliche Wadenkrämpfe nach einem Medikament verordnet wird, das

\section{Tabelle 1}

Risiko von Wadenkrämpfen nach Verordnung der genannten Medikamente

\begin{tabular}{l|l|}
$\begin{array}{l}\text { Langwirksame Beta-2- } \\
\text { Sympathomimetika }\end{array}$ & 2,42 -fach \\
\hline $\begin{array}{l}\text { Monotherapie } \\
\text { In Kombination mit } \\
\text { Steroiden }\end{array}$ & 2,17 -fach \\
\hline \begin{tabular}{l} 
Diuretika \\
\hline Kaliumsparende \\
Diuretika
\end{tabular} & 1,47 -fach \\
\hline Thiaziddiuretika & 2,12-fach \\
\hline Schleifendiuretika & $1,48-f a c h$ \\
\hline Statine & $1,20-f a c h$ \\
\hline
\end{tabular}

Ursache der Wadenkrämpfe sein könnte. Die Verordnungen wurden der Datenbank in British Columbia entnommen.

Innerhalb von fünf Jahren wurde Chinin 1590-mal nach Diuretika, 1326mal nach Statinen und 576-mal nach langwirksamen Beta-2-Sympathikomimetika verordnet. Diese drei Medikamente bzw. teils auch ihre Untergruppen führten gehäuft zur Verordnung von Chinin (s. Tab.). Unter Berücksichtigung der Kontrollgruppen - umgekehrte Reihenfolge der Verordnungen - führten damit alle drei Medikamente zu nächtlichen Wadenkrämpfen, am häufigsten langwirksame Beta-2-Sympathikomimetika (2,42-fach), gefolgt von Diuretika (1,47-fach) und Statinen (1,16-fach).

\section{- S. R. Garrison et al.}

Nocturnal leg cramps and prescription use that precedes them. Arch. Intern. Med. 172 (2012) $120-126$

\section{Kommentar}

Nächtliche Wadenkrämpfe haben bei älteren Personen eine Prävalenz von 37-50\%. Patienten werden durch diese schmerzhaften Ereignisse immer wieder aus dem Schlaf gerissen. Vorbeugung und Therapie bleiben unbefriedigend: Chinin hat viele Nebenwirkungen und Kontraindikationen, die Gabe von Magnesium hat keine gesicherte bzw. zuverlässige Wirkung. Umso wichtiger ist es, mögliche Ursachen zu eruieren. Die vorliegende Studie zeigt, dass langwirksame Beta-2-Sympathikomimetika, verschiedene
Diuretika und die Statine nächtliche Wadenkrämpfe verursachen können. Etwa $60 \%$ aller Patienten der Studie, denen Chinin verordnet wurde, hatten zuvor eines dieser drei Medikamente erhalten. Somit scheint die Mehrheit der pharmakologischen Ursachen für Wadenkrämpfe identifiziert zu sein. Wenn diese Nebenwirkung unter den genannten Medikamenten auftritt, müssen deren Indikation und evtl. Alternativen überprüft werden.

H. HOLZGREVE — 